\title{
CPV Predictions from Flavour Symmetries
}

\section{Ivan Girardi}

SISSA/INFN, Trieste, Italy

E-mail: igirardi@sissa.it

\section{S. T. Petcov*}

SISSA/INFN, Trieste, Italy and Kavli IPMU, Kashiwa, Japan

E-mail: petcovesissa.it

\section{Arsenii Titov ${ }^{\dagger}$}

SISSA/INFN, Trieste, Italy

E-mail: atitovesissa.it

In the reference 3-neutrino mixing scheme leptonic $\mathrm{CP}$ violation can be caused by the Dirac, $\delta$, and/or Majorana, $\alpha_{21}$ and $\alpha_{31}$, CP-violating phases present in the unitary neutrino mixing matrix $U$. Using the fact that $U=U_{e}^{\dagger} U_{v}, U_{e}$ and $U_{v}$ being unitary matrices diagonalising the charged lepton and neutrino mass matrices, respectively, we consider in a systematic way forms of $U_{e}$ and $U_{v}$ allowing us to derive sum rules for $\cos \delta, \alpha_{21}$ and $\alpha_{31}$, i.e., to express them in terms of the measured neutrino mixing angles present in $U$ and the angles (and phases in the case of sum rules for $\alpha_{21}$ and $\alpha_{31}$ ) contained in $U_{v}$, whose values are fixed by a discrete flavour symmetry (a generalised CP symmetry). We consider several concrete forms of $U_{v}$, including the bimaximal, tri-bimaximal and golden ratio mixing forms. For each of these forms and forms of $U_{e}$ allowing to reproduce the measured values of the neutrino mixing angles, we obtain numerical predictions for $\delta, \alpha_{21}$ and $\alpha_{31}$. Using the obtained values of the CP-violating phases, we present predictions for the effective Majorana mass in neutrinoless double beta decay.

Neutrino Oscillation Workshop

4 - 11 September, 2016

Otranto (Lecce, Italy)

*Also at: Institute of Nuclear Research and Nuclear Energy, Bulgarian Academy of Sciences, Sofia, Bulgaria.

$\dagger$ Speaker. 
Understanding the origin of the observed pattern of neutrino mixing and establishing the status of the CP symmetry in the lepton sector are among the highest priority goals of the programme of future research in neutrino physics (see, e.g., [1]). In the reference 3-neutrino mixing scheme with three light massive neutrinos (see, e.g., [1]), which we assume to be Majorana particles, CPviolating (CPV) effects can be caused by one Dirac and two Majorana CPV phases present in the Pontecorvo, Maki, Nakagawa, Sakata (PMNS) neutrino mixing matrix.

In the approach we will follow, which leads to predictions for the CPV phases, one exploits the fact that the PMNS matrix $U=U_{e}^{\dagger} U_{v}=\tilde{U}_{e}^{\dagger} \Psi \tilde{U}_{v} Q_{0}$ [2], where $U_{e}$ and $U_{v}$ are $3 \times 3$ unitary matrices originating from the diagonalisation, respectively, of the charged lepton and neutrino mass matrices. The matrices $\tilde{U}_{e}$ and $\tilde{U}_{v}$ are CKM-like $3 \times 3$ unitary matrices, and $\Psi=\operatorname{diag}\left(1, e^{-i \psi}, e^{-i \omega}\right)$ and $Q_{0}=\operatorname{diag}\left(1, e^{i \xi_{21} / 2}, e^{i \xi_{31} / 2}\right)$. It is further assumed that, up to sub-leading perturbative corrections (and phase matrices), the PMNS matrix $U$ has a specific known form $\tilde{U}_{v}$ that is dictated by a discrete flavour symmetry. Widely discussed symmetry forms of $\tilde{U}_{v}$ include: (i) bimaximal (BM) form, (ii) tri-bimaximal (TBM) form, (iii) golden ratio type A (GRA) form, (iv) golden ratio type B (GRB) form, and (v) hexagonal (HG) form (see the corresponding references in [3]). For all these forms the matrix $\tilde{U}_{v}$ represents a product of two orthogonal matrices $R_{23}$ and $R_{12}$ describing rotations in the 2-3 and 1-2 planes on fixed angles $\theta_{23}^{v}$ and $\theta_{12}^{v}$, with $\theta_{23}^{v}=-45^{\circ}$ and $\theta_{12}^{v}=45^{\circ}, \sin ^{-1}(1 / \sqrt{3}) \approx 35^{\circ}, \tan ^{-1}(1 / r) \approx 32^{\circ}(r$ being the golden ratio, $r=(1+\sqrt{5}) / 2)$, $\cos ^{-1}(r / 2)=36^{\circ}$, and $30^{\circ}$, for the BM, TBM, GRA, GRB, and HG forms, respectively.

For certain forms of $\tilde{U}_{e}$ which allow one to reproduce the measured values of the neutrino mixing angles $\theta_{12}, \theta_{13}$ and $\theta_{23}$, the Dirac CPV phase $\delta$ satisfies sum rules $[3,4,5]$ by which it is expressed in terms of $\theta_{i j}$ and the angles in $\tilde{U}_{v}$ whose values are fixed. The Majorana phases $\alpha_{21}$ and $\alpha_{31}$ similarly obey sum rules by which they are expressed in terms of the angles $\theta_{i j}$ and those of $\tilde{U}_{v}$ and the phases $\xi_{21,31}[3,6]$. In the present contribution we recapitulate the sum rules for $\cos \delta, \alpha_{21}$ and $\alpha_{31}$ derived in $[3,5,6]$ for $\tilde{U}_{v}=R_{23}\left(\theta_{23}^{v}\right) R_{12}\left(\theta_{12}^{v}\right)$ and $\tilde{U}_{e}$ being $R_{12}^{-1}\left(\theta_{12}^{e}\right)$ (case A1), $R_{13}^{-1}\left(\theta_{13}^{e}\right)$ (case A2), $R_{23}^{-1}\left(\theta_{23}^{e}\right) R_{12}^{-1}\left(\theta_{12}^{e}\right)$ (case $\mathrm{B} 1$ ) and $R_{23}^{-1}\left(\theta_{23}^{e}\right) R_{13}^{-1}\left(\theta_{13}^{e}\right)$ (case $\mathrm{B} 2$ ). All these cases can be realised when a discrete flavour symmetry group is fully broken in the charged lepton sector and is broken to a residual symmetry in the neutrino sector which fixes the matrix $\tilde{U}_{v}$ (see, e.g., [7]).

In Table 1 we present the sum rules for $\cos \delta$ in all the cases considered. It is worth noting that these sum rules are exact within the schemes considered. We give also the expressions for $s_{23}^{2} \equiv \sin ^{2} \theta_{23}$ for the schemes in which it is fixed by the values of $\theta_{13}$ and $\theta_{23}^{v}$. In Table 2 we present the sum rules for the Majorana phases $\alpha_{21}$ and $\alpha_{31}$. In this table $U$ is assumed to be in the standard parametrisation, and the corresponding sum rules for $\cos \delta$ and $s_{23}^{2}$, if any, from Table 1 should be used in each case. The phases $\varkappa_{21}$ and $\varkappa_{31}$ take values 0 or $\pi$ and are known once the angles $\theta_{i j}^{v}$ are fixed in all the cases, but B1 and B2 in which $\varkappa_{31}$ remains free. The expressions for $\varkappa_{21}$ and $\varkappa_{31}$ in terms of $\theta_{i j}^{v}$ can be found in Table 1 of [6].

We show in Table 3 the predictions for $\delta=2 \pi-\cos ^{-1}(\cos \delta)$ and the corresponding values of $\left(\alpha_{21} / 2-\xi_{21} / 2\right)$ and $\left(\alpha_{31} / 2-\xi_{31} / 2\right)\left(\left(\alpha_{31} / 2-\xi_{31} / 2-\beta\right)\right.$ in cases B1 and B2, $\beta$ being a free phase parameter, see [3, 6]) for all the schemes considered using the current best fit values of the neutrino mixing parameters $s_{12}^{2}, s_{13}^{2}$ and $s_{23}^{2}$ obtained for the neutrino mass spectrum with normal ordering (NO) in [8]. Certainly, $\delta=\cos ^{-1}(\cos \delta)$ and the corresponding values of $\left(\alpha_{21(31)} / 2-\xi_{21(31)} / 2\right)$, which are equal to $2 \pi$ minus the values in the table, are also predicted by the sum rules, but in this case $\delta$ lies in the interval $[0, \pi)$, which is almost entirely disfavoured by the global data at 


\begin{tabular}{|lll|}
\hline Case & $s_{23}^{2}$ & $\cos \delta$ \\
\hline A1 & $\frac{s_{23}^{\nu 2}-s_{13}^{2}}{1-s_{13}^{2}}$ & $\frac{\left(c_{13}^{2}-c_{23}^{\nu 2}\right)^{\frac{1}{2}}}{\sin 2 \theta_{12} s_{13}\left|c_{23}^{v}\right|}\left[\cos 2 \theta_{12}^{v}+\left(s_{12}^{2}-c_{12}^{\nu 2}\right) \frac{s_{23}^{\nu 2}-\left(1+c_{23}^{\nu 2}\right) s_{13}^{2}}{c_{13}^{2}-c_{23}^{\nu 2}}\right]$ \\
A2 & $\frac{s_{23}^{\nu 2}}{1-s_{13}^{2}}$ & $-\frac{\left(c_{13}^{2}-s_{23}^{\nu 2}\right)^{\frac{1}{2}}}{\sin 2 \theta_{12} s_{13}\left|s_{23}^{\nu}\right|}\left[\cos 2 \theta_{12}^{v}+\left(s_{12}^{2}-c_{12}^{\nu 2}\right) \frac{c_{23}^{\nu 2}-\left(1+s_{23}^{\nu 2}\right) s_{13}^{2}}{c_{13}^{2}-s_{23}^{\nu 2}}\right]$ \\
B1 & Not fixed & $\frac{\tan \theta_{23}}{\sin 2 \theta_{12} s_{13}}\left[\cos 2 \theta_{12}^{v}+\left(s_{12}^{2}-c_{12}^{\nu 2}\right)\left(1-\cot ^{2} \theta_{23} s_{13}^{2}\right)\right]$ \\
B2 & Not fixed & $-\frac{\cot \theta_{23}}{\sin 2 \theta_{12} s_{13}}\left[\cos 2 \theta_{12}^{v}+\left(s_{12}^{2}-c_{12}^{\nu 2}\right)\left(1-\tan ^{2} \theta_{23} s_{13}^{2}\right)\right]$ \\
\hline
\end{tabular}

Table 1: Sum rules for $s_{23}^{2}$ and $\cos \delta$ in cases A and B (see the text). (From [3, 5].)

\begin{tabular}{|lll|}
\hline Cases & $\alpha_{21} / 2$ & $\alpha_{31} / 2$ \\
\hline A1, B1 & $\arg \left(U_{\tau 1} U_{\tau 2}^{*} e^{i \frac{\alpha_{21}}{2}}\right)+\varkappa_{21}+\xi_{21} / 2$ & $\arg \left(U_{\tau 1}\right)+\varkappa_{31}+\xi_{31} / 2$ \\
A2, B2 & $\arg \left(U_{\mu 1} U_{\mu 2}^{*} e^{i \frac{\alpha_{21}}{2}}\right)+\varkappa_{21}+\xi_{21} / 2$ & $\arg \left(U_{\mu 1}\right)+\varkappa_{31}+\xi_{31} / 2$ \\
\hline
\end{tabular}

Table 2: Sum rules for the Majorana phases $\alpha_{21}$ and $\alpha_{31}$ in cases A and B (see the text). (From [3, 6].)

\begin{tabular}{|l|rrr|rrr|rrr|rrr|}
\hline Case & \multicolumn{3}{|c|}{ TBM } & \multicolumn{3}{|c|}{ GRA } & \multicolumn{3}{|c|}{ GRB } & \multicolumn{3}{c|}{ HG } \\
\hline A1 & 258 & 18 & 192 & 283 & 19 & 193 & 253 & 17 & 192 & 295 & 18 & 193 \\
A2 & 282 & 342 & 168 & 257 & 341 & 167 & 287 & 343 & 168 & 245 & 342 & 167 \\
B1 & 260 & 20 & 14 & 282 & 21 & 15 & 255 & 19 & 13 & 293 & 20 & 15 \\
B2 & 285 & 345 & 350 & 256 & 344 & 349 & 291 & 346 & 350 & 242 & 345 & 349 \\
\hline
\end{tabular}

Table 3: The predicted values of $\delta$ (1st sub-column), $\left(\alpha_{21} / 2-\xi_{21} / 2\right)$ (2nd sub-column) and $\left(\alpha_{31} / 2-\xi_{31} / 2\right)$ $\left(\left(\alpha_{31} / 2-\xi_{31} / 2-\beta\right)\right.$ for cases B1 and B2) (3rd sub-column) in degrees using the current best fit values of $s_{12}^{2}, s_{13}^{2}$ and $s_{23}^{2}$ for the NO neutrino mass spectrum obtained in [8]. (From [6].)

more than $2 \sigma$ level [8]. In the case of the BM symmetry form of $\tilde{U}_{v}$, the corresponding sum rules give unphysical values of $\cos \delta$, and hence of the Majorana-type phase differences, if one uses the current best fit values of $s_{12}^{2}, s_{13}^{2}$ and $s_{23}^{2}$ (for details see [3, 4]).

The results of a statistical analysis of the predictions for $\cos \delta$ have been thoroughly discussed in $[5,9,10]$, including also the impact of the renormalisation group corrections [11]. They show that a rather precise measurement of $\cos \delta$ would allow one to distinguish between the different symmetry forms of $\tilde{U}_{v}$ considered by us, thus, opening the possibility to test the discrete symmetry origin of the observed pattern of neutrino mixing.

In [6] we have shown that the requirement of generalised CP invariance of the neutrino Majorana mass term in the cases of the $A_{4}, T^{\prime}, S_{4}$ and $A_{5}$ lepton flavour symmetries leads to $\xi_{21(31)}=0$ or $\pi$. When the phases $\xi_{21}$ and $\xi_{31}$ are fixed, the values of the Majorana phases $\alpha_{21}$ and $\alpha_{31}$ are known (see Tables 2 and 3), except for cases B where $\alpha_{31}$ remains unconstrained.

As an example, in Fig. 1 we show the absolute value of the effective Majorana mass $\langle m\rangle \equiv$ $\sum_{i} m_{i} U_{e i}^{2}=m_{1} c_{12}^{2} c_{13}^{2}+m_{2} s_{12}^{2} c_{13}^{2} e^{i \alpha_{21}}+m_{3} s_{13}^{2} e^{i\left(\alpha_{31}-2 \delta\right)}, m_{1,2,3}$ being the light Majorana neutrino masses, in neutrinoless double beta $\left((\beta \beta)_{0 v^{-}}\right)$decay versus the lightest neutrino mass $m_{\min }$ in case B1 for neutrino mass spectra with NO and inverted ordering (IO) setting $\left(\xi_{21}, \xi_{31}\right)=(0,0)$ 

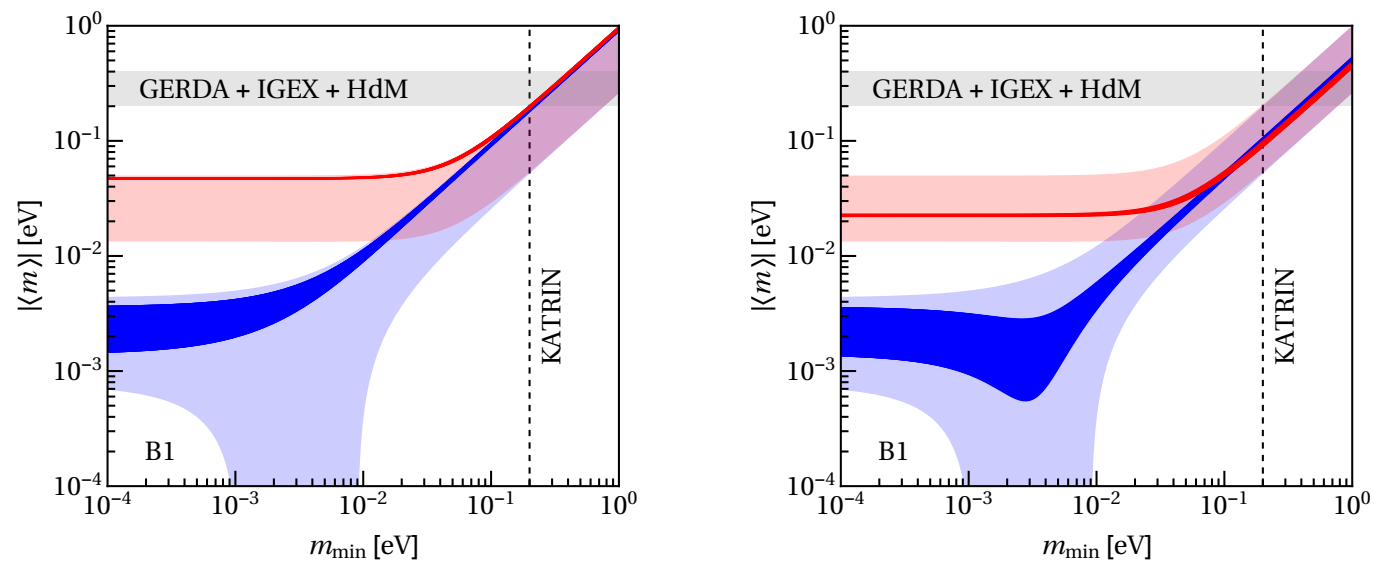

Figure 1: The effective Majorana mass $|\langle m\rangle|$ versus the lightest neutrino mass $m_{\min }$ in case $\mathrm{B} 1$ for NO (blue band) and IO (dark-red band) using the best fit values of $\theta_{12}, \theta_{13}, \Delta m_{21}^{2}$ and $\Delta m_{31(23)}^{2}$ for NO (IO) [8], and the predicted values of the CPV phases for $\left(\xi_{21}, \xi_{31}\right)=(0,0)$ (left panel) and $(\pi, 0)$ (right panel). (From [6].)

and $(\pi, 0)$. The predicted values of $|\langle m\rangle|$ for all the symmetry forms considered are within the blue (NO) and dark-red (IO) bands obtained varying the free phase $\beta$ within the interval $[0, \pi)$. The light-blue and light-red areas are obtained varying $\theta_{12}, \theta_{13}, \Delta m_{21}^{2}$ and $\Delta m_{31(23)}^{2}$ within their respective $3 \sigma$ ranges and the phases $\alpha_{21}$ and $\left(\alpha_{31}-2 \delta\right)$ within the interval $[0,2 \pi)$. As Fig. 1 shows, the parameter space gets constrained. For $\left(\xi_{21}, \xi_{31}\right)=(0,0)$ and the IO spectrum, $|\langle m\rangle|$ has practically the maximal possible values. In the case of the NO spectrum, $|\langle m\rangle|$ is always bigger than $1.5 \times 10^{-3} \mathrm{eV}$. For $\left(\xi_{21}, \xi_{31}\right)=(\pi, 0)$ and the IO spectrum, a partial compensation between the terms in $|\langle m\rangle|$ takes place. However, $|\langle m\rangle| \gtrsim 2 \times 10^{-2} \mathrm{eV}$. The mutual compensation between the different terms in $|\langle m\rangle|$ is stronger in the case of the NO spectrum. In the interval $8 \times 10^{-4} \mathrm{eV} \lesssim m_{\min } \lesssim 5 \times 10^{-3} \mathrm{eV},|\langle m\rangle| \lesssim 10^{-3} \mathrm{eV}$.

A.T. would like to thank the organisers of the Neutrino Oscillation Workshop 2016 for their kind invitation and the opportunity to present this work.

\section{References}

[1] K. Nakamura and S. T. Petcov in C. Patrignani et al. (PDG), Chin. Phys. C 40 (2016) 100001.

[2] P. H. Frampton, S. T. Petcov and W. Rodejohann, Nucl. Phys. B 687 (2004) 31.

[3] S. T. Petcov, Nucl. Phys. B 892 (2015) 400.

[4] D. Marzocca, S. T. Petcov, A. Romanino and M. C. Sevilla, JHEP 1305 (2013) 073.

[5] I. Girardi, S. T. Petcov and A. V. Titov, Eur. Phys. J. C 75 (2015) 345.

[6] I. Girardi, S. T. Petcov and A. V. Titov, Nucl. Phys. B 911 (2016) 754.

[7] I. Girardi, S. T. Petcov, A. J. Stuart and A. V. Titov, Nucl. Phys. B 902 (2016) 1.

[8] F. Capozzi, E. Lisi, A. Marrone, D. Montanino and A. Palazzo, Nucl. Phys. B 908 (2016) 218.

[9] I. Girardi, S. T. Petcov and A. V. Titov, Nucl. Phys. B 894 (2015) 733.

[10] I. Girardi, S. T. Petcov and A. V. Titov, Int. J. Mod. Phys. A 30 (2015) 1530035.

[11] J. Gehrlein, S. T. Petcov, M. Spinrath and A. V. Titov, JHEP 1611 (2016) 146. 